\title{
Peak reduction technique in commutative algebra
}

\author{
Vladimir Shpilrain ${ }^{\dagger}$ \\ and
}

Jie-Tai Yu*

\begin{abstract}
The "peak reduction" method is a powerful combinatorial technique with applications in many different areas of mathematics as well as theoretical computer science. It was introduced by Whitehead, a famous topologist and group theorist, who used it to solve an important algorithmic problem concerning automorphisms of a free group. Since then, this method was used to solve numerous problems in group theory, topology, combinatorics, and probably in some other areas as well.

In this paper, we give a survey of what seems to be the first applications of the peak reduction technique in commutative algebra and affine algebraic geometry. Using this technique, we have contributed toward a classification of two-variable polynomials having classified, up to an automorphism, polynomials of the form $a x^{n}+b y^{m}+\sum_{i m+j n \leq m n} c_{i j} x^{i} y^{j}$ (i.e., polynomials whose Newton polygon is either a triangle or a line segment). This has several applications to the study of embeddings of algebraic curves in the plane. In particular, upon combining our method with a well-known theorem of Zaidenberg and Lin, we have shown that one can decide "almost" just by inspection whether or not a polynomial fiber $\{p(x, y)=0\}$ is an irreducible simply connected curve in $\mathbf{C}^{2}$.

Recently, P.Wightwick used the idea of peak reduction in combination with splice diagrams technique due to D.Eisenbud and W.Neumann to classify all twovariable polynomials over $\mathbf{C}$ up to an automorphism.

Another application that we present here, yields a decomposition of the group $\operatorname{Aut}(K[x, y])$ in a free product with amalgamation.
\end{abstract}

\section{Introduction}

In this paper, we present what seems to be the first applications of the "peak reduction" method due to Whitehead (see [15] or [7]) in commutative algebra and affine algebraic geometry. In general, this method is used to find some kind of canonical form of a given object $P$ under the action of a given group (or a semigroup) $T$ of transformations.

The general idea behind this method is rather simple: one chooses the complexity of an object $P$ one way or another, and declares a canonical form of $P$ an object

1991 Mathematics Subject Classification: Primary 14E09, 14E25; Secondary 13B10, 13B25.

$\dagger$ †) Partially supported by CRCG Grant 25500/301/01.

*) Partially supported by Hong Kong RGC Grant Project 7126/98P. 
$P^{\prime}$ whose complexity is minimal among all objects $t(P), t \in T$. To actually find a "canonical model" $P^{\prime}$ of a given object $P$, one tries to arrange a sequence of sufficiently simple transformations so that the complexity of an object decreases at every step. To prove that such an arrangement is possible, one uses "peak reduction"; that means, if in some sequence of simple transformations the complexity goes up (or remains unchanged) before eventually going down, then there must be a pair of subsequent simple transformations in the sequence (a "peak") such that one of them increases the maximum degree (or leaves it unchanged), and then the other one decreases it. Then one tries to prove that such a peak can always be reduced.

In commutative algebra context, objects are polynomials; their complexity is their degree; the group of transformations is the group of polynomial automorphisms; simple transformations are elementary and linear automorphisms. (An elementary automorphism is a one that changes just one variable).

More specific details on this technique are given in the end of the Introduction and in Sections 2 and 3. Here we start by presenting results obtained by using this method. We also point out that, although this technique was originally created for solving an algorithmic problem, it can be also used to obtain some structural descriptions - we illustrate this in Section 3.

Let $K[x, y]$ be the polynomial algebra in two variables over a field $K$ of characteristic 0 . The main motivation of our paper [14] was the following problem, which is similar to the problem considered by Whitehead in the context of a free group:

Problem 1. Given two polynomials $p, q \in K[x, y]$, find out if there is an automorphism of $K[x, y]$ that takes $p$ to $q$.

In [14], we contributed toward a solution of this problem by establishing the following

Theorem 1.1. 14] Let $p(x, y)=a x^{n}+b y^{m}+\sum_{i m+j n \leq m n} c_{i j} x^{i} y^{j}, a, b, c_{i j} \in K, i, j>0$; $a, b \neq 0$, and $q(x, y)=A x^{r}+B y^{s}+\sum_{i s+j r \leq r s} b_{i j} x^{i} y^{j}, A, B, b_{i j} \in K, i, j>0 ; A, B \neq 0$. Suppose that $m$ does not divide $n, n$ does not divide $m, s$ does not divide $r, r$ does not divide $s$, and $\max (m, n) \neq \max (r, s)$. Then there is no automorphism $\alpha \in A u t(K[x, y])$ that takes $p(x, y)$ to $q(x, y)$.

In some special cases, we can handle those polynomials with $m$ divisible by $n$ or vice versa. This is possible, for example, if some fiber of a given polynomial admits a one-variable polynomial parametrization $x=u(t) ; y=v(t)$ :

Proposition 1.2. [14] Suppose the fibers $\{p(x, y)=0\},\{q(x, y)=0\}$ of two polynomials $p, q \in \mathbf{C}[x, y]$, admit one-variable polynomial parametrizations. Then one can effectively find out (even without knowing the parametrizations) if there is an automorphism of $\mathbf{C}[x, y]$ that takes $p$ to $q$.

In particular, if some fiber of a given polynomial is an irreducible simply connected curve, then, by a well-known theorem of Zaidenberg and Lin [18, this fiber admits 
a one-variable polynomial parametrization. More precisely, they prove that (in case $K=\mathbf{C}$ ) every polynomial like that has a "canonical model" of the form $x^{k}-y^{l}$ with $(k, l)=1$. (This generalizes earlier result of Abhyankar and Moh [1]). Upon combining this with our method, we have the following

Theorem 1.3. [14] Let $p(x, y) \in \mathbf{C}[x, y]$ be a polynomial whose fiber $\{p(x, y)=0\}$ is an irreducible simply connected curve. Then some automorphism of $\mathbf{C}[x, y]$ takes $p(x, y)$ to $x^{k}-y^{l}$ with $(k, l)=1$, and:

(a) $\max (k, l) \leq \operatorname{deg}(p(x, y))$;

(b) either $k$ or $l$ divides $\operatorname{deg}(p(x, y))$;

(c) the Newton polygon of $p(x, y)$ is either a triangle or a line segment, i.e., $p(x, y)$ is of the form $a x^{n}+b y^{m}+\sum_{i m+j n \leq m n} c_{i j} x^{i} y^{j}, i, j>0, a, b \neq 0$. If $m$ does not divide $n$, $n$ does not divide $m$, and $m, n \neq 0$, then $m=k$ or $l$, and $n=l$ or $k$, respectively. Otherwise, either $p(x, y)$ is linear, or the "leading" part $a x^{n}+b y^{m}+\sum_{i m+j n=m n} c_{i j} x^{i} y^{j}$ is a proper power of some other polynomial.

Thus, in many situations it is possible to rule out polynomials without irreducible simply connected fibers just by inspection. In any case, by Proposition 1.2, there is an effective procedure for deciding if a given polynomial fiber is irreducible and simply connected.

Recently, Wightwick [16] used the idea of peak reduction in combination with splice diagrams technique (see [6]) to completely solve Problem 1 for two-variable polynomials over C. The key ingredient of her solution is the following

Theorem 1.4. [16], [9] Let $p \in \mathbf{C}[x, y]$ be a non-constant polynomial and $\phi \in$ $\operatorname{Aut}(\mathbf{C}[x, y])$. Then there is a factorization of $\phi$ in a product $\phi_{1} \phi_{2} \ldots \phi_{n}$ of elementary and linear automorphisms, such that, for $p_{i}=\phi_{1} \phi_{2} \ldots \phi_{i}(p), i=1, \ldots, n$, and $p_{0}=p$, one has:

$$
\operatorname{deg}\left(p_{0}\right) \geq \ldots \geq \operatorname{deg}\left(p_{k}\right) \leq \ldots \leq \operatorname{deg}\left(p_{n}\right), \text { for some } 0 \leq k \leq n .
$$

Moreover, there is at most one $\phi_{i}$ for which $\operatorname{deg}\left(p_{i-1}\right)=\operatorname{deg}\left(p_{i}\right)$, and if this happens then $\operatorname{deg}\left(p_{i}\right)$ is the minimal degree $\operatorname{deg}\left(p_{k}\right)$.

This result provides a procedure for finding a "canonical model" for a given polynomial $p$ (i.e., an automorphic image of $p$ whose degree cannot be reduced by any automorphism). Indeed, given a polynomial $p$, we check if there is an elementary automorphism that reduces the degree of $p$. This actually amounts to checking automorphisms of the form $\left\{x \rightarrow x, y \rightarrow \lambda_{1} \cdot y+\lambda_{2} \cdot x^{k}\right\}$ and $\left\{x \rightarrow \lambda_{1} \cdot x+\lambda_{2} \cdot y^{k}, y \rightarrow y\right\}$, with $k \leq \operatorname{deg}(p)$. To check if an elementary automorphism like that (for a particular $k$ ) can reduce the degree of $p$, one has to find out if a specific system of polynomial 
equations for $\lambda_{1}, \lambda_{2}$ has a solution. The latter can be done by using Gröbner basis technique (see e.g. [2]). If no elementary automorphism can reduce the degree, then we already have a canonical model - this is precisely the point of Theorem 1.4.

What is left now to complete the solution of Problem 1, is to be able to decide whether or not a canonical model for a given polynomial $p$ can be taken to a canonical model for another given polynomial $q$ by a sequence of elementary and linear automorphisms of $\mathbf{C}[x, y]$ ), none of which changes the degree of $p$. This, again, ultimately comes down to deciding whether or not a specific system of polynomial equations over $\mathbf{C}$ has a solution.

We now briefly describe our method that was used to prove Theorem 1.1, Proposition 1.2, and Theorem 1.3, leaving the details to the following sections.

It is a well-known result of Jung and van der Kulk that every automorphism of $K[x, y]$ is a product of elementary and linear automorphisms. We want to get a canonical model for a given polynomial by finding a sequence of elementary and linear automorphisms that would reduce the degree at every step, until it is further irreducible by any elementary automorphism. Then this last polynomial, whose degree is irreducible, will be a canonical model.

To arrange that, we use two principal ideas. First, we mimic elementary automorphisms of $K[x, y]$ by "elementary transformations" of $K[t] \times K[t]$. Second, we use Whitehead's idea of "peak reduction" (see e.g. [0]) to arrange a sequence of elementary transformations of $K[t] \times K[t]$ so that the maximum degree would decrease at every step. This means the following. If at some point of a sequence of ET, the maximum degree goes up (or remains unchanged) before eventually going down, then there must be a pair of subsequent ET in our sequence (a "peak") such that one of them increases the maximum degree (or leaves it unchanged), and then the other one decreases it. We show that such a peak can always be reduced. This is described in the next Section 2 .

While the "peak reduction" always works for elementary transformations of $K[t] \times$ $K[t]$, the first part (mimicking elementary automorphisms of $K[x, y]$ by elementary transformations of $K[t] \times K[t])$ is where the difficulty is. We managed to do that for polynomials of the form given in Theorem 1.1, and also for polynomials $p(x, y)$ whose fiber $\{p(x, y)=0\}$ admits a one-variable polynomial parametrization $x=u(t) ; y=v(t)$ (i.e., this fiber is a rational curve with one place at infinity). The latter was used in proving Proposition 1.2 and Theorem 1.3.

It would be interesting and important to find applications of the peak reduction method to the study of the group $\operatorname{Aut}(K[x, y, z])$, or, at least, of the subgroup of this group generated by tame automorphisms (those are products of elementary and linear automorphisms).

A progress in this direction could lead to proving some particular automorphisms of $K[x, y, z]$ to be non-tame, thus resolving a well-known problem due to Nagata (see e.g. [3]). In particular, one can ask: 
Problem 2. Let $p=p(x, y, z) \in K[x, y, z]$. Is it true that if the degree of $p$ can be decreased by a sequence of elementary and linear automorphisms, then it can also be decreased by a single elementary automorphism?

To conclude the Introduction, we mention some earlier results that were originally established by different methods, but can be re-proved in a uniform way by using the peak reduction technique:

(1) 10] An algorithm for deciding whether or not a given matrix from $G L_{2}(K[x, y])$ is a product of elementary and diagonal matrices.

(2) 112 An algorithm for deciding whether or not a given polynomial from $K[x, y]$ is a coordinate, i.e., an automorphic image of $x$. This was later generalized in [5] to detecting coordinates in $K[z][x, y]$.

(3) A decomposition of the group $\operatorname{Aut}(K[x, y])$ in a free product with amalgamation. Several decompositions of this group have been previously known; see our Section 3 for details. Also in Section 3, we use the peak reduction method to obtain a decomposition which is slightly different from the previously known ones.

\section{Elementary automorphisms and peak reduction}

We give here a somewhat more precise statement of a well-known result of Jung and van der Kulk which can be found in [3, Theorem 6.8.5]:

Proposition 2.1. Every automorphism of $K[x, y]$ is a product of linear automorphisms and automorphisms of the form $x \rightarrow x+f(y) ; y \rightarrow y$. More precisely, if $\left(g_{1}, g_{2}\right)$ is an automorphism of $K[x, y]$ such that $\operatorname{deg}\left(g_{1}\right) \geq \operatorname{deg}\left(g_{2}\right)$, say, then either $\left(g_{1}, g_{2}\right)$ is a linear automorphism, or there exists a unique $\mu \in K^{*}$ and a positive integer $d$ such that $\operatorname{deg}\left(g_{1}-\mu g_{2}^{d}\right)<\operatorname{deg}\left(g_{1}\right)$.

Now we are going to consider the direct product $K[t] \times K[t]$ of two copies of the one-variable polynomial algebra over $K$, and introduce the following elementary transformations (ET) that can be applied to elements of this algebra:

(ET1) $(u, v) \longrightarrow\left(u+\mu \cdot v^{k}, v\right)$ for some $\mu \in K^{*} ; k \geq 2$.

(ET2) $(u, v) \longrightarrow\left(u, v+\mu \cdot u^{k}\right)$.

(ET3) a non-degenerate linear transformation $(u, v) \longrightarrow\left(a_{1} u+a_{2} v, b_{1} u+b_{2} v\right)$; $a_{1}, a_{2}, b_{1}, b_{2} \in K$.

One might notice that some of these transformations are redundant, e.g., (ET1) is a composition of the other ones. There is a reason behind that which will be clear a little later.

Our proof of Theorem 1.1 was based on the following

Proposition 2.2. 14] For any pair $(u, v) \in K[t] \times K[t]$, there is a (perhaps, empty) sequence of elementary transformations that takes $(u, v)$ to some $(\hat{u}, \hat{v})$ such that: 
(i) the maximum of the degrees of polynomials decreases at every step in this sequence;

(ii) the maximum of the degrees in $(\hat{u}, \hat{v})$ is irreducible by any sequence of elementary transformations.

Comment to (i): if it happens so that $u$ and $v$ have the same leading terms, then, perhaps by somewhat abusing the language, we say that the transformation $(u, v) \rightarrow$ $(u-v, v)$ reduces the maximum of the degrees.

We give a proof of Proposition 2.2 here as a sample of our technique.

Proof. We shall use the "peak reduction" method to prove this statement. In this context, this means the following. If at some point of a sequence of ET, the maximum degree goes up (or remains unchanged) before eventually going down, then there must be a pair of subsequent ET in our sequence (a "peak") such that one of them increases the maximum degree (or leaves it unchanged), and then the other one decreases it. We are going to show that such a peak can always be reduced. In other words, if the maximum degree can be decreased by a sequence of ET, then it can also be decreased by a single ET. To prove that, we have to consider many different cases, but all of them are quite simple.

Let $(u, v)$ be a pair of polynomials from $K[t] \times K[t]$ with, say, $\operatorname{deg}(u) \leq \operatorname{deg}(v)$, and let $\alpha_{1}$ and $\alpha_{2}$ be two subsequent ET applied to $(u, v)$, as described in the previous paragraph. Consider several cases:

(1) $\alpha_{1}:(u, v) \longrightarrow\left(u+\mu \cdot v^{k}, v\right)$ for some $\mu \in K^{*} ; k \geq 2$.

This $\alpha_{1}$ strictly increases the maximum degree $\operatorname{since} \operatorname{deg}(u) \leq \operatorname{deg}(v)$ by the assumption. Now we have two possibilities for $\alpha_{2}$ since a linear ET cannot decrease the maximum degree in this situation.

(a) $\alpha_{2}:\left(u+\mu \cdot v^{k}, v\right) \longrightarrow\left(u+\mu \cdot v^{k}, v+\lambda\left(u+\mu \cdot v^{k}\right)^{m}\right)$ for some $\lambda \in K^{*} ; m \geq 2$. But this obviously increases the maximum degree, contrary to our assumption.

(b) $\alpha_{2}:\left(u+\mu \cdot v^{k}, v\right) \longrightarrow\left(u+\mu \cdot v^{k}+\lambda \cdot v^{m}, v\right)$. If this $\alpha_{2}$ decreases the maximum degree, then we should have $\mu \cdot v^{k}=-\lambda \cdot v^{m}$, in which case $\alpha_{2}=\alpha_{1}^{-1}$, and the peak reduction is just cancelling out $\alpha_{1}$ and $\alpha_{2}$.

(2) $\alpha_{1}:(u, v) \longrightarrow\left(u, v+\mu \cdot u^{k}\right)$ for some $\mu \in K^{*} ; k \geq 2$.

If this $\alpha_{1}$ increases the maximum degree, this can only happen when $\operatorname{deg}(v+\mu$. $\left.u^{k}\right)=\operatorname{deg}\left(u^{k}\right)$, in which case we argue exactly as in the case (1). However, since $\operatorname{deg}(u) \leq \operatorname{deg}(v)$, it might happen that this $\alpha_{1}$ does not change the maximum degree. Then we consider two possibilities for $\alpha_{2}$ :

(a) $\alpha_{2}:\left(u, v+\mu \cdot u^{k}\right) \longrightarrow\left(u, v+\mu \cdot u^{k}+\lambda \cdot u^{m}\right)$. If this $\alpha_{2}$ decreases the maximum degree, then we should have $m \geq k$. If $m=k$, then $\alpha_{1} \alpha_{2}$ is equal to a single ET. If $m>k$, then, in order for $\alpha_{2}$ to decrease the maximum degree, we must have $\operatorname{deg}(v)$ divisible by $\operatorname{deg}(u)$, in which case $\alpha_{2}$ alone would decrease the maximum degree of $(u, v)$, i.e., we can get rid of $\alpha_{1}$. 
(b) $\alpha_{2}:\left(u, v+\mu \cdot u^{k}\right) \longrightarrow\left(u+\lambda\left(v+\mu \cdot u^{k}\right)^{m}, v+\mu \cdot u^{k}\right)$. But this $\alpha_{2}$ can only change the degree of the first polynomial in the pair, and this is not where the maximum degree was.

(3) $\alpha_{1}$ is linear, i.e., $\alpha_{1}:(u, v) \longrightarrow\left(a_{1} u+a_{2} v, b_{1} u+b_{2} v\right) ; a_{1}, a_{2}, b_{1}, b_{2} \in K$. Again, we have two possibilities for $\alpha_{2}$.

(a) $\alpha_{2}:\left(a_{1} u+a_{2} v, b_{1} u+b_{2} v\right) \longrightarrow\left(a_{1} u+a_{2} v, b_{1} u+b_{2} v+\mu\left(a_{1} u+a_{2} v\right)^{k}\right)$. If $k=1$, then $\alpha_{2}$ is linear, and therefore $\alpha_{1} \alpha_{2}$ is a single ET. If $k>1$, then $\alpha_{2}$ might decrease the maximum degree, but this can only happen if $a_{2}=0$, in which case we could decrease the maximum degree of $(u, v)$ by a single ET of the type (ET2).

(b) the case where $\alpha_{2}$ is of the type (ET1), is completely similar.

Thus, in each of the considered cases, if there is a "peak", then we can reduce the number of ET in the sequence. An obvious inductive argument completes the proof of Proposition 2.2.

\section{Decomposing the group of polynomial automorphisms}

In this section, we give an application of the "peak reduction" method to produce a new decomposition of the group $A u t(K[x, y])$ in a free product with amalgamation.

There is a decomposition of the group $\operatorname{Aut}(K[x, y])$ in a free product with amalgamation due to Shafarevich [11]; see also [3, Theorem 6.8.6], [- [1, [17] and references thereto. In 13], we offered a somewhat more peculiar decomposition.

To describe our new decomposition and to compare it to previously known ones, we have to introduce some more notation.

We denote:

- by Af the group of affine automorphisms of $K[x, y]$;

- by $U T$ the group of upper triangular automorphisms; those are automorphisms of the form $x \rightarrow a x+p(y) ; y \rightarrow b y+c$, where $a, b, c \in K ; p(y) \in K[y]$.

- by $L T$ the group of lower triangular automorphisms; those are automorphisms of the form $x \rightarrow a x+b ; y \rightarrow c y+p(x)$.

- by TUT the group of "twisted" upper triangular automorphisms; those are automorphisms of the form $x \rightarrow a x+p(y) ; y \rightarrow b y+c x+d$, where $a, b, c, d \in K ; p(y) \in K[y]$.

- by TLT the group of "twisted" lower triangular automorphisms; those are automorphisms of the form $x \rightarrow a x+b y+c ; y \rightarrow d y+p(x)$.

Note that $T U T \cap T L T=A f$.

There are the following well-known decompositions in a free product with amalgamation:

$$
\operatorname{Aut}(K[x, y])=A f *_{A f \cap U T} U T=A f *_{A f \cap L T} L T .
$$


Our decomposition has a more symmetric form:

Theorem 3.1. Aut $(K[x, y])=T U T *_{T U T \cap T L T} T L T=T U T *_{A f} T L T$.

This cannot be claimed as a brand new result; although it probably does not appear anywhere else in exactly this form, it can be easily deduced from (1), as well as (1) can be easily deduced from Theorem 1.3. Our proof however is new and basically selfcontained; we only use the aforementioned Jung-van der Kulk theorem, whereas all known proofs of (1) also use Nagao's theorem [8]. Crucial for the proof is the following Lemma 3.2; we give a proof of this lemma here, as another sample of our method.

We are going to distinguish non-linear automorphisms within the groups TUT and $T L T$. To this end, we introduce the following elementary transformations (ET) applied to pairs of polynomials from $K[x, y]$ :

(E1) $(u, v) \longrightarrow\left(u+a \cdot v^{k}, v\right)$ for some $a \in K^{*} ; k \geq 2$.

(E2) $(u, v) \longrightarrow\left(u, v+a \cdot u^{k}\right)$.

(E3) non-degenerate affine transformations $(u, v) \longrightarrow\left(a_{1} u+a_{2} v+c_{1}, b_{1} u+b_{2} v+\right.$ $\left.c_{2}\right) ; a_{1}, a_{2}, b_{1}, b_{2}, c_{1}, c_{2} \in K$.

These elementary transformations generate a group which is isomorphic to $A u t(K[x, y])$, by the theorem of Jung and van der Kulk. Now comes

Lemma 3.2. The group generated by transformations of the type (E1) and (E2) is a free product of the subgroup generated by the transformations (E1) and the one generated by the transformations (E2). (Note that both these subgroups are abelian).

Proof is again based on the "peak reduction" method. In this context, this means the following. If at some point of a sequence of ET (of the type (E1) or (E2)), the maximum degree goes up (or remains unchanged) before eventually going down, then there must be a pair of subsequent ET in our sequence (a "peak") such that one of them increases the maximum degree (or leaves it unchanged), and then the other one decreases it. We are going to show that such a peak can always be reduced.

Let $(u, v)$ be a pair of polynomials from $K[x, y]$ with, $\operatorname{say,} \operatorname{deg}(u) \leq \operatorname{deg}(v)$, and let $\gamma_{1}$ and $\gamma_{2}$ be two subsequent ET applied to $(u, v)$, as described in the previous paragraph. Consider several cases:

(1) $\gamma_{1}:(u, v) \longrightarrow\left(u+a \cdot v^{k}, v\right)$ for some $a \in K^{*} ; k \geq 2$.

This $\gamma_{1}$ strictly increases the maximum degree since $\operatorname{deg}(u) \leq \operatorname{deg}(v)$ by the assumption. Now we have two possibilities for $\gamma_{2}$ :

(a) $\gamma_{2}:\left(u+\mu \cdot v^{k}, v\right) \longrightarrow\left(u+\mu \cdot v^{k}, v+b\left(u+\mu \cdot v^{k}\right)^{m}\right)$ for some $b \in K^{*} ; m \geq 2$. But this obviously increases the maximum degree, contrary to our assumption.

(b) $\gamma_{2}:\left(u+\mu \cdot v^{k}, v\right) \longrightarrow\left(u+\mu \cdot v^{k}+b \cdot v^{m}, v\right)$. If this $\gamma_{2}$ decreases the maximum degree, then we should have $a \cdot v^{k}=-b \cdot v^{m}$, in which case $\gamma_{2}=\gamma_{1}^{-1}$, and the peak reduction is just cancelling out $\gamma_{1}$ and $\gamma_{2}$. 
(2) $\gamma_{1}:(u, v) \longrightarrow\left(u, v+a \cdot u^{k}\right)$ for some $a \in K^{*} ; k \geq 2$.

If this $\gamma_{1}$ increases the maximum degree, this can only happen when $\operatorname{deg}(v+a$. $\left.u^{k}\right)=\operatorname{deg}\left(u^{k}\right)$, in which case we argue exactly as in the case (1). However, since $\operatorname{deg}(u) \leq \operatorname{deg}(v)$, it might happen that this $\gamma_{1}$ does not change the maximum degree. Then we consider two possibilities for $\gamma_{2}$ :

(a) $\gamma_{2}:\left(u, v+a \cdot u^{k}\right) \longrightarrow\left(u, v+a \cdot u^{k}+b \cdot u^{m}\right)$. If this $\gamma_{2}$ decreases the maximum degree, then we should have $m \geq k$. If $m=k$, then $\gamma_{1} \gamma_{2}$ is equal to a single ET. If $m>k$, then, in order for $\gamma_{2}$ to decrease the maximum degree, we must have $\operatorname{deg}(v)$ divisible by $\operatorname{deg}(u)$, in which case $\gamma_{2}$ alone would decrease the maximum degree of $(u, v)$, i.e., we can switch $\gamma_{1}$ and $\gamma_{2}$ (note that these transformations commute since they are both of the type (E2)).

(b) $\gamma_{2}:\left(u, v+a \cdot u^{k}\right) \longrightarrow\left(u+b\left(v+a \cdot u^{k}\right)^{m}, v+a \cdot u^{k}\right)$. But this $\gamma_{2}$ can only change the degree of the first polynomial in the pair, and this is not where the maximum degree was.

Thus, given a product of ET of the type (E1) or (E2), we can, after possibly cancelling pairs of successive ET of the form $\gamma^{-1} \gamma$ and switching successive ET of the same type, get another product of ET (representing, of course, the same automorphism as the given one, call it $\tau$ ), where each factor decreases the maximum degree of a pair of polynomials, starting with the pair $(\tau(x), \tau(y))$, and ending up with the pair $(x, y)$. At every step, the choice of ET that can decreases the maximum degree, is unique.

Therefore, for any automorphism from the group generated by transformations of the type (E1) and (E2), there is a unique alternating product of automorphisms from the subgroup generated by the transformations (E1) and the one generated by the transformations (E2). This completes the proof of Lemma 3.2.

\section{References}

[1] S. S. Abhyankar and T.-T. Moh, Embeddings of the line in the plane, J. Reine Angew. Math. 276 (1975), 148-166.

[2] W. Adams and P. Loustaunau, An introduction to Gröbner bases. American Mathematical Society, Providence, 1994.

[3] P. M. Cohn, Free Rings and Their Relations. 2nd Ed. Academic Press, London, 1985.

[4] W. Dicks, Automorphisms of the polynomial ring in two variables, Publ. Sec. Mat. Univ. Autonoma Barcelona 27 (1983), 155-162.

[5] V. Drensky and J.-T. Yu, Tame and wild coordinates of $K[z][x, y]$, Trans. Amer. Math. Soc., to appear.

[6] D. Eisenbud and W.D. Neumann, Three-dimensional link theory and invariants of plane curve singularities. Ann. Math. Stud. 110, Princeton. Princeton Univ. Press (1985). 
[7] R. Lyndon and P. Schupp, Combinatorial Group Theory. Series of Modern Studies in Math. 89. Springer-Verlag, 1977.

[8] H. Nagao, On GL(2, $K[x])$, J. Inst. Polytech. Osaka City Univ. Ser. A 10 (1959), 117-121.

[9] W.D. Neumann and P. Wightwick, Algorithms for polynomials in two variables, this volume.

[10] H. Park, A Computational Theory of Laurent Polynomial Rings and Multidimensional FIR Systems, Ph.D. Thesis, Univ. of California, Berkeley, 1995.

[11] I. R.Shafarevich, On some infinite-dimensional groups, Rend. Mat. e Appl. 25 (1966), 208-212.

[12] V. Shpilrain and J.-T. Yu, Polynomial automorphisms and Gröbner reductions, J. Algebra 197 (1997), 546-558.

[13] V. Shpilrain and J.-T. Yu, On generators of polynomial algebras in two commuting or non-commuting variables, J. Pure Appl. Algebra 132 (1998), 309-315.

[14] V. Shpilrain and J.-T. Yu, Embeddings of curves in the plane, J. Algebra 217 (1999), 668-678.

[15] J.H.C.Whitehead, On equivalent sets of elements in a free group, Ann. of Math. 37 (1936), 782-800.

[16] P.G. Wightwick, Polynomial automorphisms, preprint.

[17] D. Wright, The amalgamated free product structure of $G L_{2}\left(k\left[X_{1}, \ldots, X_{n}\right]\right)$ and the weak Jacobian theorem for two variables, J. Pure Appl. Algebra 12 (1978), $235-251$.

[18] M.G.Zaidenberg, V.Ya.Lin, An irreducible, simply connected algebraic curve in $\mathbf{C}^{2}$ is equivalent to a quasihomogeneous curve, Soviet Math. Dokl. 28 (1983), 200-204.

Department of Mathematics, The City College of New York, New York, NY 10031

e-mail address: shpil@groups.sci.ccny.cuny.edu

Department of Mathematics, The University of Hong Kong, Pokfulam Road, Hong Kong

e-mail address: yujt@hkusua.hku.hk 